University of Wollongong

Research Online

Faculty of Engineering and Information

Faculty of Engineering and Information

Sciences - Papers: Part B

Sciences

2019

Effects of grain boundary on wear of graphene at the nanoscale: A molecular dynamics study

Jie Zhang

Tsinghua University, jz248@uowmail.edu.au

Xinchun Chen

Tsinghua University

Qiang Xu

Tsinghua University

Tianbao Ma

Tsinghua University

Yuanzhong $\mathrm{Hu}$

Tsinghua University

See next page for additional authors

Follow this and additional works at: https://ro.uow.edu.au/eispapers1

Part of the Engineering Commons, and the Science and Technology Studies Commons

Research Online is the open access institutional repository for the University of Wollongong. For further information contact the UOW Library: research-pubs@uow.edu.au 


\title{
Effects of grain boundary on wear of graphene at the nanoscale: A molecular dynamics study
}

\author{
Abstract \\ Graphene has shown excellent tribological behaviors, enabling its potential applications as lubricating \\ and anti-wear coatings, however, the grain boundaries (GBs) formed during the preparation process may \\ deteriorate the performance of graphene. Using large-scale molecular dynamics simulations, we study \\ the wear mechanism of graphene GBs with various misorientation angles between two grains. Compared \\ with pure nanoindentation at the GBs, the critical load of wear failure upon nanoscratching across the \\ GBs is much lower due to the synergetic actions of interlocking and pushing between the tip and \\ graphene atoms. The misorientation angle between the adjacent grains significantly effects the onset and \\ fashion of atomic-scale wear. Results show that wear resistance of the graphene with large-angle GBs is \\ slightly lower than that of pristine graphene. Nevertheless, a number of the long bonds emerge in the \\ vicinity of the low-angle GBs during scratching, leading to wear failure at much smaller load than the \\ large-angle GBs. Furthermore, wear resistance of the low-angle GBs can be enhanced by increasing the \\ interfacial strength between graphene and substrate due to the reduced number of the long bonds at the \\ GB. This study sheds light on improving wear resistance of graphene coating by properly controlling its \\ microstructures.

\section{Disciplines} \\ Engineering | Science and Technology Studies

\section{Publication Details} \\ Zhang, J., Chen, X., Xu, Q., Ma, T., Hu, Y., Wang, H., Tieu, A. Kiet. \& Luo, J. (2019). Effects of grain boundary \\ on wear of graphene at the nanoscale: A molecular dynamics study. Carbon, 143 578-586.

\section{Authors} \\ Jie Zhang, Xinchun Chen, Qiang Xu, Tianbao Ma, Yuanzhong Hu, Hui Wang, Anh Kiet Tieu, and Jianbin Luo
}


Effects of grain boundary on wear of graphene at the nanoscale: a molecular dynamics study

Jie Zhang ${ }^{1}$, Xinchun Chen ${ }^{1}$, Qiang $\mathrm{Xu}^{1}$, Tianbao $\mathrm{Ma}^{1, *}$, Yuanzhong $\mathrm{Hu}^{1}$, Hui Wang ${ }^{1}$, A. Kiet Tieu $^{2}$, Jianbin Luo ${ }^{1, *}$

${ }^{1}$ State Key Laboratory of Tribology, Tsinghua University, Beijing, 100084, China

${ }^{2}$ School of Mechanical, Materials and Mechatronic Engineering, University of Wollongong, Wollongong, NSW 2522, Australia

\begin{abstract}
Graphene has shown excellent tribological behaviors, enabling its potential applications as lubricating and anti-wear coatings, however, the grain boundaries (GBs) formed during the preparation process may deteriorate the performance of graphene. Using large-scale molecular dynamics simulations, we study the wear mechanism of graphene GBs with various misorientation angles between two grains. Compared with pure nanoindentation at the GBs, the critical load of wear failure upon nanoscratching across the GBs is much lower due to the
\end{abstract}

*Corresponding author. Tel: +86-010-62788310. E-mail: mtb@mail.tsinghua.edu.cn (Tianbao Ma)

*Corresponding author. Tel: +86-010-62781385. E-mail: 1uojb@mail.tsinghua.edu.cn (Jianbin Luo) 
synergetic actions of interlocking and pushing between the tip and graphene atoms. The misorientation angle between the adjacent grains significantly effects the onset and fashion of atomic-scale wear. Results show that wear resistance of the graphene with large-angle GBs is slightly lower than that of pristine graphene. Nevertheless, a number of the long bonds emerge in the vicinity of the low-angle GBs during scratching, leading to wear failure at much smaller load than the large-angle GBs. Furthermore, wear resistance of the low-angle GBs can be enhanced by increasing the interfacial strength between graphene and substrate due to the reduced number of the long bonds at the GB. This study sheds light on improving wear resistance of graphene coating by properly controlling its microstructures.

\section{Introduction}

Graphene is an atomically thin layer of $\mathrm{sp}^{2}$ covalently bonded carbon atoms packed into a honeycomb lattice. Due to its remarkable mechanical [1-3], lubricating [4-6], and most recently superlubricity properties [7-9], graphene has the potential applications to serve as anti-wear material and coating for micro- and nanoelectromechanical systems (MEMS/NEMS). Considerable endeavors have been made to investigate nanoscale wear mechanism of graphene by atomic force microscopy (AFM) experiments and molecular dynamics (MD) simulations. Vasić et al. $[10,11]$ attributed wear of graphene to the graphene edge, where graphene is easily peeled-off with increased lateral force due to stable wrinkles formation. Qi et al. showed that due to the atom-by-atom adhesive wear and peeling induced rupture, the graphene step edge 
shows much weaker wear resistance, lowering the load carrying capacity of graphene by 2 orders of magnitudes as compared with that in the interior region [12]. The wear resistance of the step edge can be improved at humid environment as the dangling bonds of carbon atoms are passivated [13]. Atomic roughness of the contacting surfaces can induce inhomogeneous local contact pressure fluctuations, which also lead to a decline in wear resistance of graphene $[14,15]$. Klemenz et al. proposed that graphene coating on the indenter can mitigate the effect of atomic-scale indenter roughness and eliminate its influence on nanoindentation measurement [16]. An effective way of suppressing such an inhomogeneous pressure distribution and enhancing wear-resistance of graphene is to coat graphene on both sides of the sliding interface as predicted by MD simulations [14], which has been verified by experiments $[17,18]$. Thickness is another factor influencing the anti-wear performance of coatings. In general, the increasing number of graphene layers can mitigate the local stress fluctuations, improving wear resistance of graphene coating [19]. Vasić et al. [19] argued based on their AFM experiments that a minimum of $5 \mathrm{~nm}$ (roughly 15 layers) thickness is necessary for wear protection. Stone-Wales (SW) type defects and vacancy type defects also play important roles in tribological behaviors of graphene. Sun et al [20] showed that higher potential barriers induced by SW or vacancy defect on the surface layer of graphene can result in the increase of friction. Moreover, vacancy defect induces the exposure of dangling bonds, which may lead to severe wear [14].

As growing large-area single-crystalline graphene is still a big challenge by chemical vapor 
deposition(CVD) [21, 22] and epitaxial growth [23], graphene coatings are usually composed of grains with different sizes and orientation angles, and grain boundaries (GBs) are formed when any adjacent grains merge during the growth [24]. The polycrystalline structure of graphene has been considered to be detrimental to the strength of graphene [25-29], so it is significant to identify the roles of grain boundaries in nanoscale wear of graphene for potential anti-wear applications. Sha et al. studied the nanoindentation of polycrystalline graphene, where the failure behaviors is found to be critically dependent on the indentation site, including grain center, grain boundary,, grain boundary triple junction, and holes [30]. Kavalur and Kim studied the influences of polycrystalline structures on the interlayer friction of graphene by MD simulations. Their results showed that the grain boundaries of polycrystalline graphene with certain misorientation angles lead to an increase of friction. However, the study on nanoscale wear mechanism of the grain boundary structure is urgently needed to provide guidelines for improving the anti-wear performance of graphene layer.

In this study, MD stimulations are carried out to investigate the roles of grain boundaries in nanoscale wear of graphene. Bicrystal graphene monolayers with representative armchair- and zigzag-oriented grain boundaries in various misorientation angles were constructed according to previous experimental and theoretical findings [31-35]. To model an AFM nanoscratching test on graphene [19], a nanoscale tip with a constant load was forced to slide on the monolayer graphene supported on the surface of $\mathrm{SiO}_{2}$. The synergetic interlocking and pushing interaction between the tip and graphene atoms during the nanoscratching process enables the breaking of 
the carbon-carbon bonds and fracture of graphene under much less normal load than pure nanoindentation. It is shown that the discrepancies of wear resistance between the low- and large-angle GBs mainly originates from the number of the long bonds around the GBs. Based on the above analysis, we propose that an increase in the interfacial strength between graphene and substrate can help mitigate the weakening effects of the grain boundaries and improve the wear resistance of polycrystalline graphene.

\section{Simulation methods}

By transmission electron microscopy (TEM) and scanning tunneling microscopy (STM) characterizations of the CVD-grown graphene [36, 37] and epitaxial graphene $[38,39]$, the GBs are found to be predominantly composed of the pentagon-heptagon pairs or the 5-7 defects with strain-induced hillocks along the GBs $[38,40]$. Following the coincidence site lattice (CSL) theory proposed by Carlsson et al [41], we generated bicrystal graphene monolayer with six representative grain boundaries (GBs) in various misorientation angles $\theta$ as shown in Fig. 1(a)(f). A series of coincidence points with a regular periodicity along a line can be found for the specified misorientation angle. Cutting the two graphene sheets along the line and removing unwanted atoms yields an initial form of a GB. The conjugate gradient method was used to minimize the potential energy and generate the optimized structures of the GB. More details with regard to the procedure of grain boundary generation can be found in Ref. [41]. In this work, the zigzag-oriented GBs in Fig.1(a)-(c) are denoted by ZZ-51, ZZ-132 and ZZ-217, 
where 51,132 and 217 correspond to the misorientation angles of $\theta=5.1^{\circ}, \theta=13.2^{\circ}$ and $\theta=$ 21.7 $7^{\circ}$ Correspondingly, the armchair-oriented GBs with the misorientation angles in Fig.1(d)(f), $\theta=17.9^{\circ}, \theta=21.8^{\circ}$ and $\theta=27.8^{\circ}$, are indicated by AC-179, AC-218 and AC-278. The pristine armchair and zigzag graphene samples are denoted by AC-0 and ZZ-0, respectively.

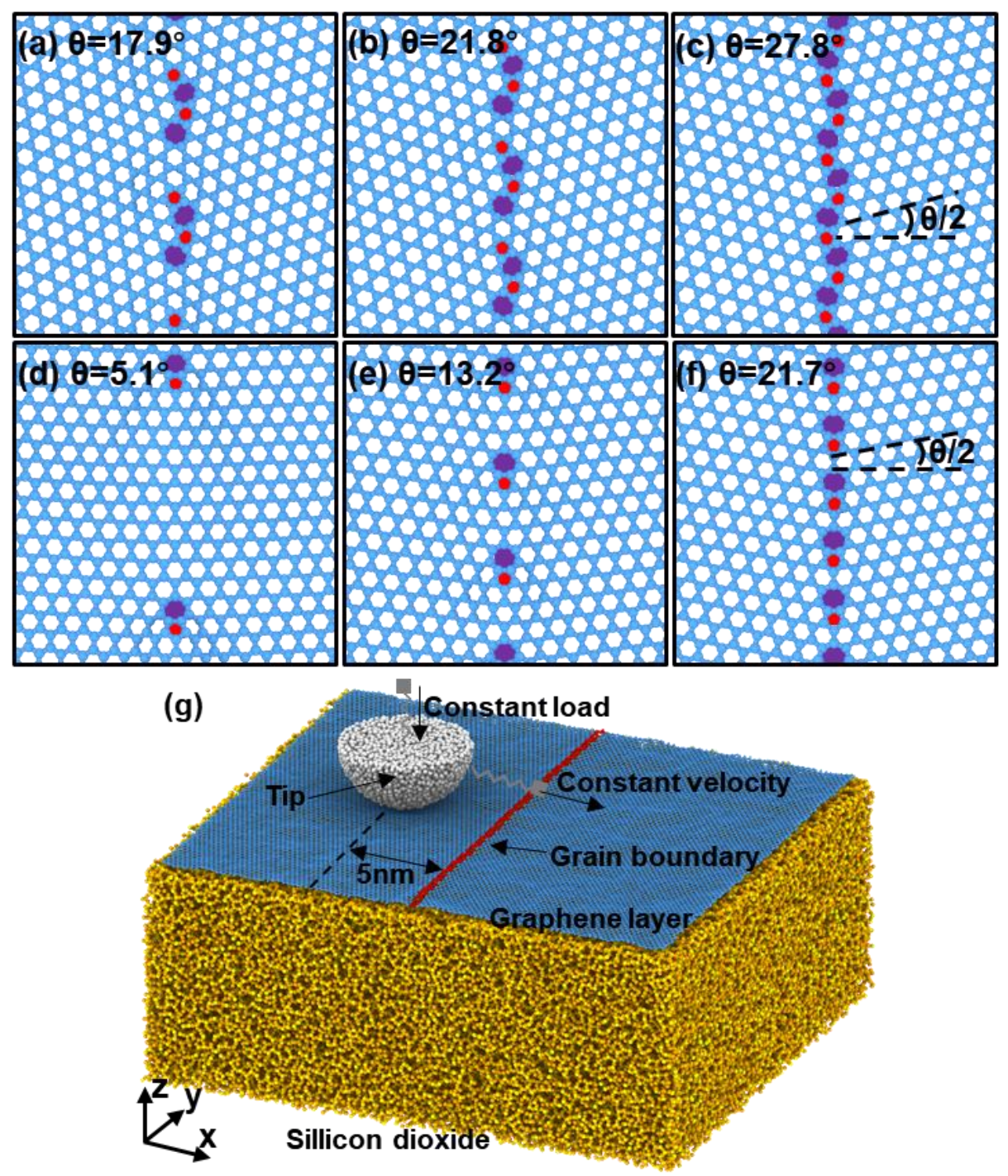

Fig.1. Atomic structures of grain boundaries in (a-c) armchair-oriented and (d-f) zigzagoriented graphene with different misorientation angles. 5- and 7-membered rings are colored in red and purple, respectively. (g) Atomistic model of a DLC tip sliding against a monolayer graphene layer with a GB supported by a $\mathrm{SiO}_{2}$ substrate. 
A schematic of our atomistic model is illustrated in Fig. 1g. In the model, a hemispherical amorphous carbon (a-C) tip with a radius of $3 \mathrm{~nm}$ is sliding against monolayer graphene with a GB supported by a deformable substrate of amorphous silicon dioxide. The tip was obtained by cutting a hemisphere from the a-C solid prepared by high-temperature quenching and rapid cooling based on a previous method [42]. The tip is treated as rigid with 8808 carbon atoms and coupled with normal and lateral springs for incorporating the cantilever compliance in an AFM system. The normal spring is used to apply constant load with a stiffness of $48 \mathrm{~N} / \mathrm{m}$, while the lateral spring is used to drag the tip along the $x$ direction with a stiffness of $64 \mathrm{~N} / \mathrm{m}$ [12]. The dimensions of the graphene sheet and the $\mathrm{SiO}_{2}$ substrate are roughly $24.4 \mathrm{~nm} \times 22.0$ $\mathrm{nm}$ in the $x$ and $y$ directions, and $25.9 \mathrm{~nm} \times 22.9 \mathrm{~nm} \times 10.5 \mathrm{~nm}$, respectively. The $\mathrm{SiO}_{2}$ substrate contains 307558 oxygen atoms and 153682 silicon atoms, respectively. For pristine graphene and GB-containing graphene layers, the number of carbon atoms ranges from 20064 to 20875 . For the scratching case, the initial distance between the tip apex and the GB are $5 \mathrm{~nm}$ along the $x$ direction. This distance is chosen to mitigate any influences on the GBs owing to the loading process before scratching. The total sliding distance of the tip is $8 \mathrm{~nm}$, which ensures that the tip scratches across the GBs completely. For the case of indenting, the tip is positioned right above the GB. Periodic boundary conditions are applied along the $x$ and $y$ directions. The atoms in the bottommost of the substrate are fixed. To constrain translational motion of the whole graphene layer, the carbon atoms at the edge are fixed along the $x$ and $y$ directions but move freely along the $z$ direction. The Nosé-Hoover thermostat was employed to the free atoms, 
maintaining a system temperature of $300 \mathrm{~K}$. The Nosé-Hoover thermostat was employed to the free atoms according to previous work $[12,13]$, maintaining a system temperature of $300 \mathrm{~K}$. It should be noted that the thermostat is typically applied only to the atoms away from the contact region in friction simulations [43]. Comparing with an alternative approach as shown in Fig. $\mathrm{S} 1$, the present thermostat scheme does not show artificial effects on the simulation results.

The interatomic interactions within graphene were modeled by a REBO2+S potential, which has been developed to accurately capture $\mathrm{C}-\mathrm{C}$ bond-breaking events by using screening functions $[42,44]$ and estimate the tensile strength of graphene [2]. A Tersoff potential with optimized parameter sets for $\mathrm{Si}-\mathrm{O}$ system [45] was used to describe the interactions within the $\mathrm{SiO}_{2}$ substrate. Interactions between graphene, $\mathrm{SiO}_{2}$ substrate and a-C tip were modeled by Lennard-Jones potentials. For C-C interactions between graphene and a-C tip, the parameters of $\sigma=0.34 \mathrm{~nm}$ and $\varepsilon=2.84 \mathrm{meV}$ were used $[14,46]$. For $\mathrm{C}-\mathrm{O}$ and $\mathrm{C}-\mathrm{Si}$, we used the parameters given in Qi's work [12], rendering the work of adhesion between graphene and the substrate comparable to the experimental data[47]. Before loading, the whole system was allowed to relax for 50ps at $300 \mathrm{~K}$ using the NVT ensemble. Due to different bond pre-strain of 5-7 defects along the GBs, the average heights of the GB buckling on the $\mathrm{SiO}_{2}$ substrate for $\mathrm{AC}-179$, $\mathrm{AC}$ 218 and AC-278 are $0.22 \mathrm{~nm}, 0.18 \mathrm{~nm}$ and $0.02 \mathrm{~nm}$, respectively. For the GBs of ZZ-51, ZZ132 and ZZ-217, the average heights are $0.26 \mathrm{~nm}, 0.22 \mathrm{~nm}$ and $0 \mathrm{~nm}$, respectively. For both indenting and scratching, the tip apex was initially placed $2 \mathrm{~nm}$ above the graphene layer, ensuring there are no interactions between the tip and the graphene layer during relaxation. A 
series of normal loads were applied with an interval of $10 \mathrm{nN}$. At each load, we relaxed the system for $10 \mathrm{ps}$. The tip was dragged by the lateral spring at a constant speed of $20 \mathrm{~m} / \mathrm{s}$ under the constant load. To evaluate the effects of scratching and indenting positions on the results, the tip was forced to scratch along three lines, and indent three points on the graphene layer as shown in Fig. S2, which yield the similar critical normal loads as shown in Table S1 and S2. Without further mention, the following results are based on scanning line 1 and indenting point 1. All simulations were performed by using LAMMPS [48].

\section{Results and discussion}

3.1 Wear mechanism of graphene with GBs

To study the effects of GBs on wear resistance of graphene, we start with scratching each GBcontaining graphene layer and identifying critical normal loads for wear failure. Here, the wear failure was characterized by the emergence of permanent breaking of $\mathrm{C}-\mathrm{C}$ bonds, according to previous definitions $[2,14,49]$. The normal loads were gradually increased by $10 \mathrm{nN}$ for each scratching until wear occurs. It has been shown that pristine mono-layer graphene supported on the substrate could lead to a reduction of friction $[2,19,50]$. Scratching on the substrate covered with the graphene layer at the normal load below $100 \mathrm{nN}$ results in extremely low friction as shown in Fig.2, which is consistent with previous report [2]. It is also shown in Fig.2 that average friction force at the normal load below $300 \mathrm{nN}$ is much lower on the GB-containing graphene layer than that on the $\mathrm{SiO}_{2}$ substrate, indicating that the GB-containing graphene may 
still act as a solid lubricant at the small load. The abrupt increase of the friction force in Fig.2 indicates breaking of $\mathrm{C}-\mathrm{C}$ bonds, rupture of graphene at the critical normal load corresponding to each GB model. From Table 1, the critical normal loads on ZZ-51 and AC-179 GBs are reduced by $24 \%$ and $27 \%$, respectively, as compared to the corresponding pristine graphene. For ZZ-217, AC-218 and AC-278 GBs with the misorientation angles larger than $20^{\circ}$, however, the critical normal loads are very close to those of pristine graphene.

Table 1. Critical normal load $\mathrm{F}_{\mathrm{c}}$ for armchair- and zigzag-orientated graphene.

\begin{tabular}{cccccccccc}
\hline & \multicolumn{4}{c}{ Armchair-oriented } & & \multicolumn{3}{c}{ Zigzag-oriented } \\
\cline { 2 - 4 } \cline { 8 - 10 } & AC-0 & AC-179 & AC-218 & AC-278 & & ZZ-0 & ZZ-51 & ZZ-132 & ZZ-217 \\
\hline $\mathrm{F}_{\mathrm{c}}(\mathrm{nN})$ & 420 & 320 & 400 & 410 & & 440 & 320 & 370 & 400 \\
\hline
\end{tabular}

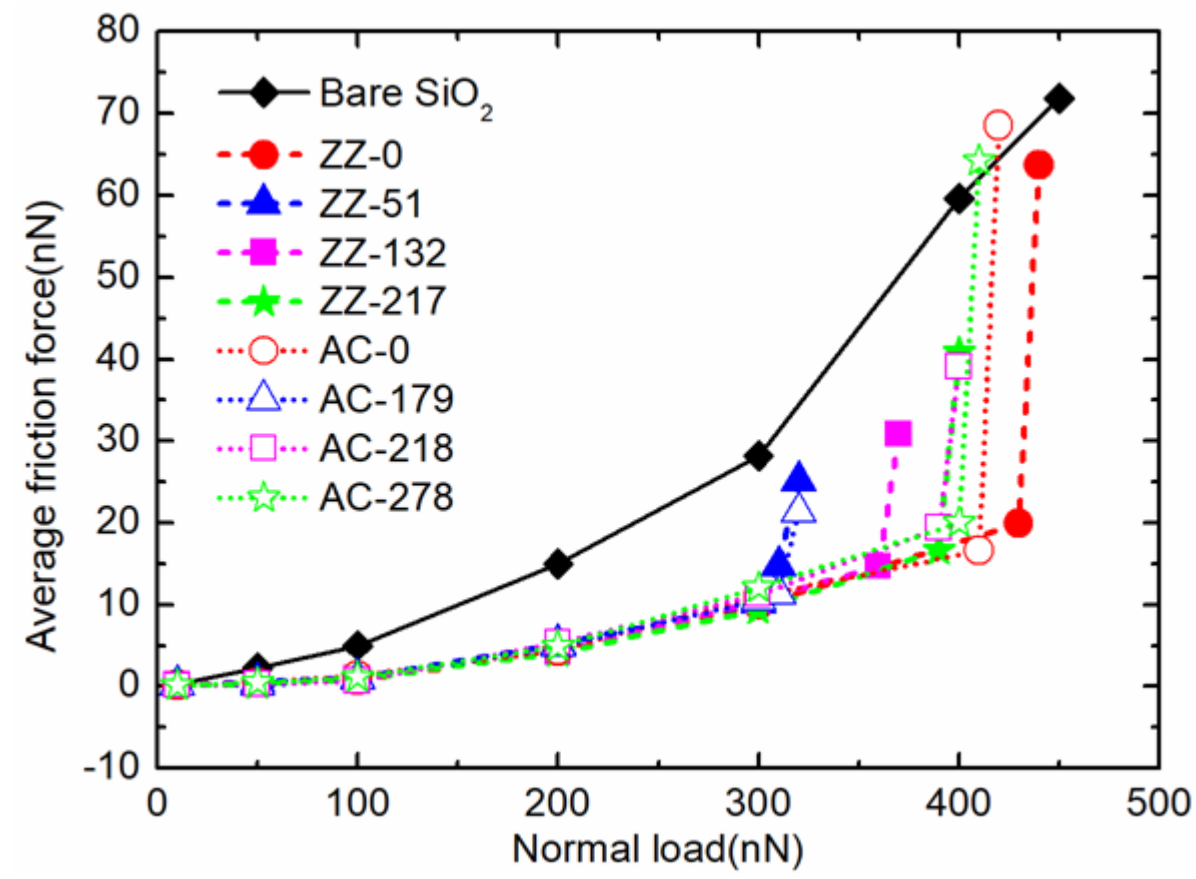

Fig.2. Average friction force as a function of normal load for scratching simulations on bare $\mathrm{SiO}_{2}$, pristine graphene and graphene with GBs. 
(a) Sliding distance $=3.75 \mathrm{~nm}$

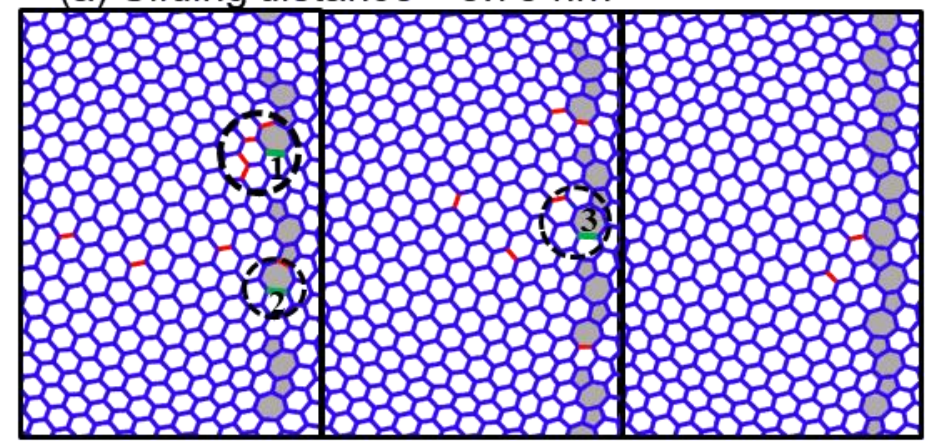

(b) Sliding distance $=4.45 \mathrm{~nm}$

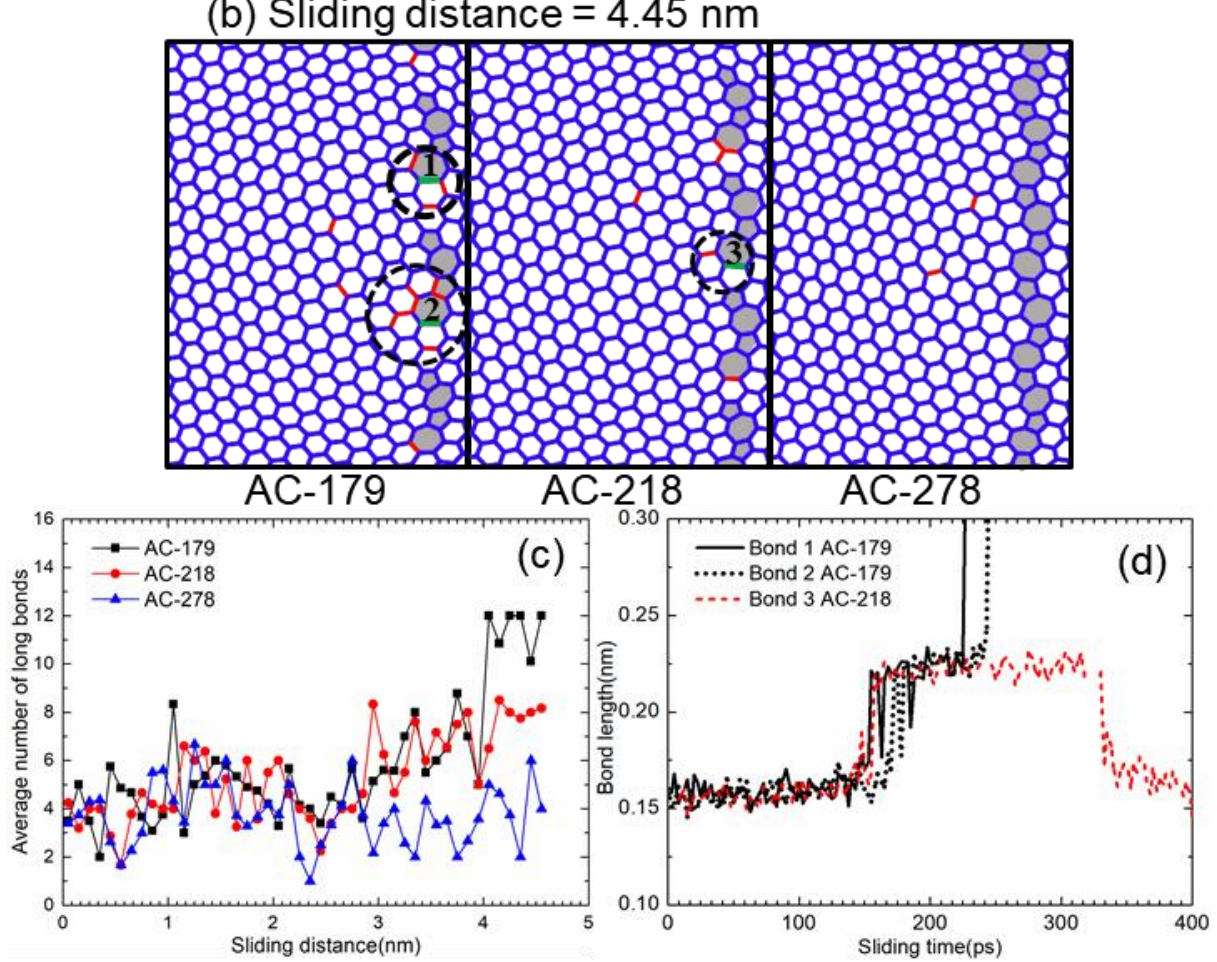

Fig.3. Variations of the long bonds at the contact region at $320 \mathrm{nN}$ for different GBs. (a-b)

Distribution of the long bonds for AC-179, AC-218 and AC-278 at two sliding distances of

$3.75 \mathrm{~nm}$ and $4.45 \mathrm{~nm}$, respectively. The critical bonds (green) are numbered, and they are encircled with their neighbor long bonds (red) by dashed lines. 5-7 defects along the GBs are colored in grey. (c) Average (within interval of $0.1 \mathrm{~nm}$ ) of the number of long bonds as a function of sliding distance before AC-179 starts to break. The tip sliding initiates at a distance of $5 \mathrm{~nm}$ towards the GB. (d) Length of bond 1-3 in (a) and (b) as a function sliding time. 
The lower strength of the low-angle GBs under the uniaxial tensile loading was ascribed to the higher pre-strain of the critical bonds shared by the heptagon and hexagon rings for a wide range of temperature and strain-rate $[31,33]$. Unlike the uniaxial tensile loading, the graphene layers here suffered from much more complex local stresses and strains under tip scratching within the contact area. Therefore, we believe that there are other mechanisms controlling angle-dependent wear failure of the GB-containing graphene layers. For better comparison, the AC-218 and AC-278 were also scratched at a normal load of $320 \mathrm{nN}$ which is the critical load for AC-179. The number of the C-C bonds with bond length longer than $0.160 \mathrm{~nm}$ in the contact area was calculated and tracked during the scratching process for AC-179, AC-218 and AC278. The critical bond length defined here is very close to the longest pre-strain bond among the relaxed GBs $(0.153 \mathrm{~nm})$. The contacting atoms of graphene were identified as those separated less than $0.4 \mathrm{~nm}$ from the tip atoms $[51,52]$. The distribution of the long bonds at two specified sliding distances were first characterized. It is shown in Fig.3a-b that most of the long bonds for AC-179 emerge at the side of the GB critical bonds. At the sliding distance of 4.45 $\mathrm{nm}$ as shown in Fig.3b, there are three and seven of neighbor long bonds for bond 1 and bond 2 of AC-179, respectively. In contrast, only one long bond is in the vicinity of bond 3 , and the distribution of the long bonds for AC-218 is relatively scattered in the contact area as shown in Fig.3b. In order to explore the roles of the GBs in the variations of the long bonds, furthermore, we calculated the average number of the long bonds during the whole scratching process. It is found in Fig 3c that the average number of the long bonds for both AC-179 and AC-218 
increases as the tip approaches the GBs, but the number of the long bonds of AC-179 becomes larger than that of AC-218 after the sliding distance reaches $4 \mathrm{~nm}$. For AC-278, there are only fluctuations in the number of the long bonds in Fig.3c. Since the C-C bond lengths of the 5-7 defects in AC-278 are comparable to those of the hexagon rings [31], AC-278 has the least effects on the variations of the long bonds during the tip scratching among the armchairoriented GBs as shown in Fig.3a-c. Based on the above analysis, failure of graphene with AC179 at $320 \mathrm{nN}$ is attributed to a number of the long bonds assembled in the vicinity of the GB critical bonds. Those long bonds enable permanent breaking of bond 1 and bond 2 as shown in Fig.3d. For AC-218, however, it is observed in Fig.3d that bond 3 reforms after the tip scratches over the GB at the same normal load, and there is no further damage. We also conducted similar analysis of the distribution and the number of the long bonds for ZZ-51 and ZZ-217, which confirms that the accumulation of "long bonds" in the vicinity of the GBs leads to the lower wear resistance of the low-angle GBs (see detail in Supporting Information section 3).

3.2 Comparison between scratching and indentation upon graphene with GBs

The distribution of the long bonds analyzed in section 3.1 can be used to understand wear resistance of the GBs with various misorientation angles. In this section, we explore the role of scratching action in nanoscale wear of GB-containing graphene. For the case of indentation shown in Fig.4a, the tip was initially placed above the GB and the stepped loads were applied until the graphene breaks. For pristine armchair-oriented and zigzag-oriented graphene, the 
breaking loads are respectively $530 \mathrm{nN}$ and $520 \mathrm{nN}$, which is close to $501 \mathrm{nN}$ predicted by previous molecular mechanics simulations [2]. In contrast, the simulation model for scratching is shown in Fig.4b. It is interesting to note that the critical normal loads of all GBs for indentation are larger than those for scratching as shown in Fig.4c and 4d. That is to say, scratching imposes a harsher working condition for graphene. For GB-containing graphene, the critical normal load for indentation increases with the misorientation angle as shown in Fig. $4 \mathrm{c}$ and $4 \mathrm{~d}$.
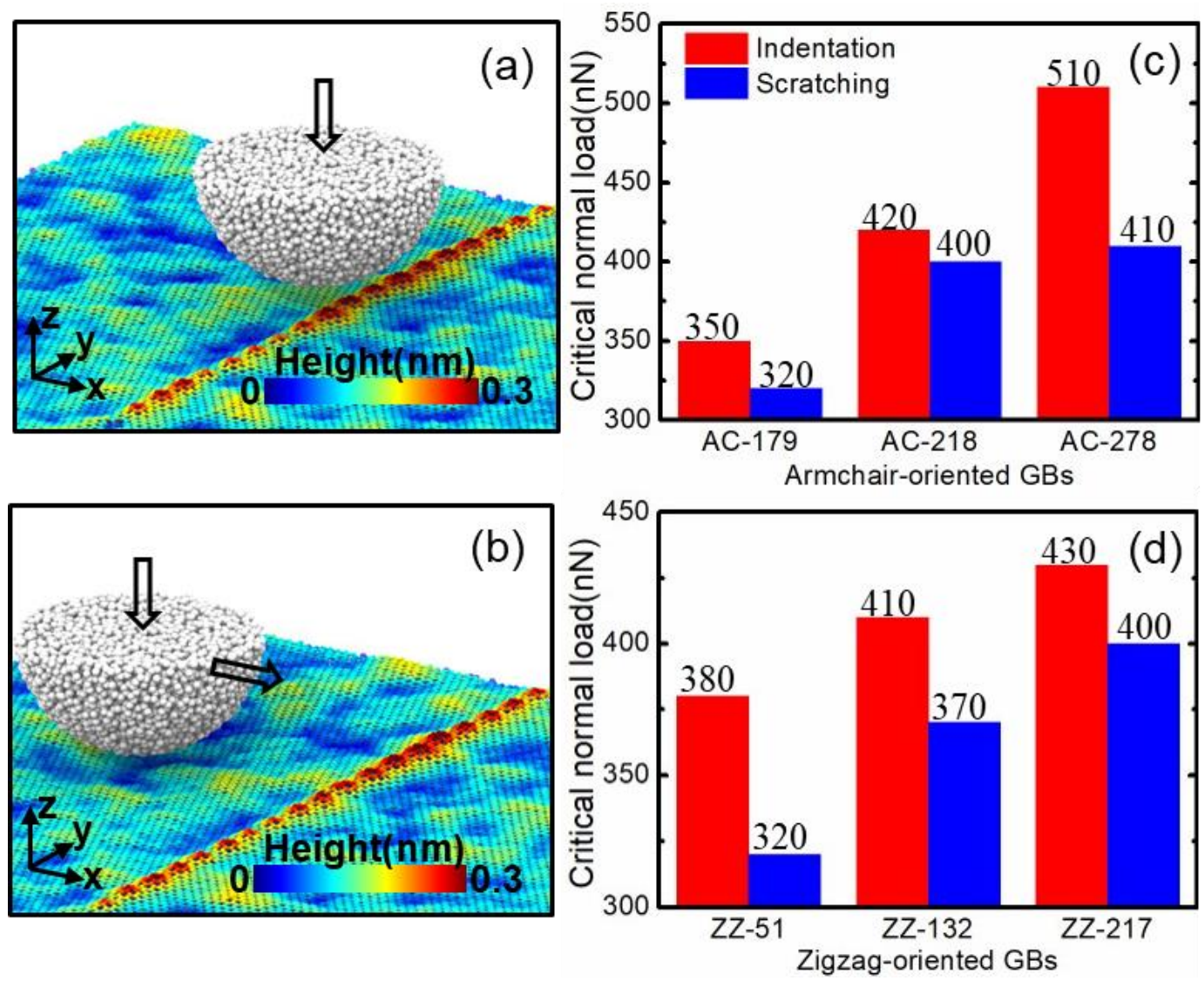

Fig.4. Schematic representations for (a) indenting upon the GB and (b) scratching across the GB. Critical normal loads of failure for (c) armchair-oriented and (d) zigzag-oriented GBs. 
As shown in Fig.5a, ZZ-132 deforms with several breaking bonds in the contact region under indentation, and the difference in the relative displacement of the atoms indicates it is stretched locally along the $x$ direction. For scratching, on the other hand, the graphene deforms plastically under the same load of indentation after the tip scratches to the position of the GB as shown in Fig.5b. In contrast with indentation, the graphene atoms in the contact region during the scratching process all displace forwards along the $\mathrm{x}$ direction due to the action of pushing from the tip atoms. It should be noted that the graphene layer also undergoes local stretching as the relative displacement of the contacting atoms are not uniform as shown in Fig.5b.

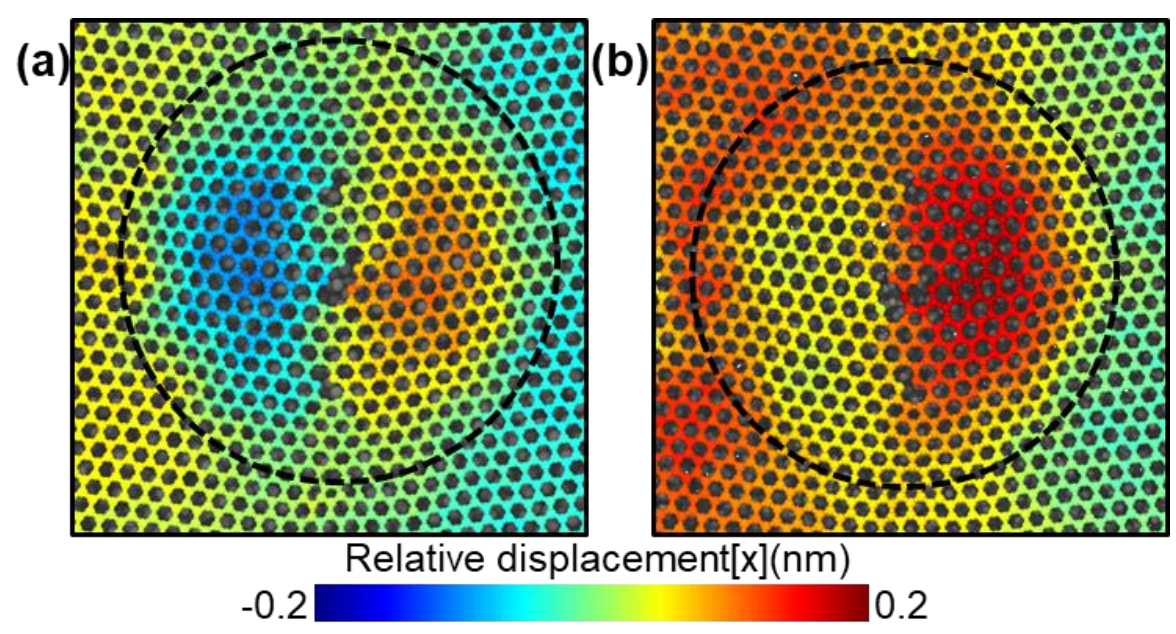

Fig.5. X-component of relative displacements of each atom with regarding to their initial positions for (a) loading normally upon ZZ-132 and (b) shearing perpendicularly across ZZ132 grain boundary both at the load of $370 \mathrm{nN}$. Dashed circles indicate the edge of the contact region between the graphene and the tip.

We found that the co-action of interlocking and pushing between the tip and graphene atoms 
during the scratching results in the discrepancy of critical normal load between scratching and indentation. The atomistic interlocking could cause high friction during sliding as the tip atoms are trapped into hollow positions of graphene $[14,46,51]$. Here, two damage mechanisms are induced by the co-action of interlocking and pushing. During the scratching process, the tip atoms will firstly fall interlocked in the graphene lattice. As shown in Fig. 6, at $t=252.0 \mathrm{ps,}$ atom $B$ of the tip is interlocked above the bond between carbon atoms 2 and 3 of the graphene layer. Then at $t=258.0 \mathrm{ps}$ atom B exerts lateral forces along the sliding direction and break the bond between atoms 2 and 3, and then move forwards. Meanwhile, atom 1 is pushed by atom $A$ with the force of $4.4 \mathrm{nN}$. The pushing forces on atom 1 and atom 2 together lead to the lateral movement of the carbon chain connecting with them and consequently the breaking of the bond nearby (indicated by black dotted circles in Fig.6). Similarly, at $t=269.2 \mathrm{ps}$, atom $C$ exerted a large force of $7.0 \mathrm{nN}$ to atom 5 , resulting in the breaking of the bond between atom 4 and atom 5. This interlocking-pushing behavior happens repeatedly during the scratching process, since the tip atoms are continuously falling into interlocking geometries at different positions of the graphene layer, posing a greater chance to break the C-C bonds of graphene. In contrast, the chances for atomic interlocking and pushing are much less for pure indentation due to the lack of interfacial sliding process. As the tip atoms may fall into different interlocking geometries with different GBs of the graphene layer, scanning along different lines and indenting at different points could produce small variations of critical normal load as shown in Table S1 and S2. The interlocking-pushing behavior also happens in armchair-oriented GB models (see 
detail in Supporting Information section 4).

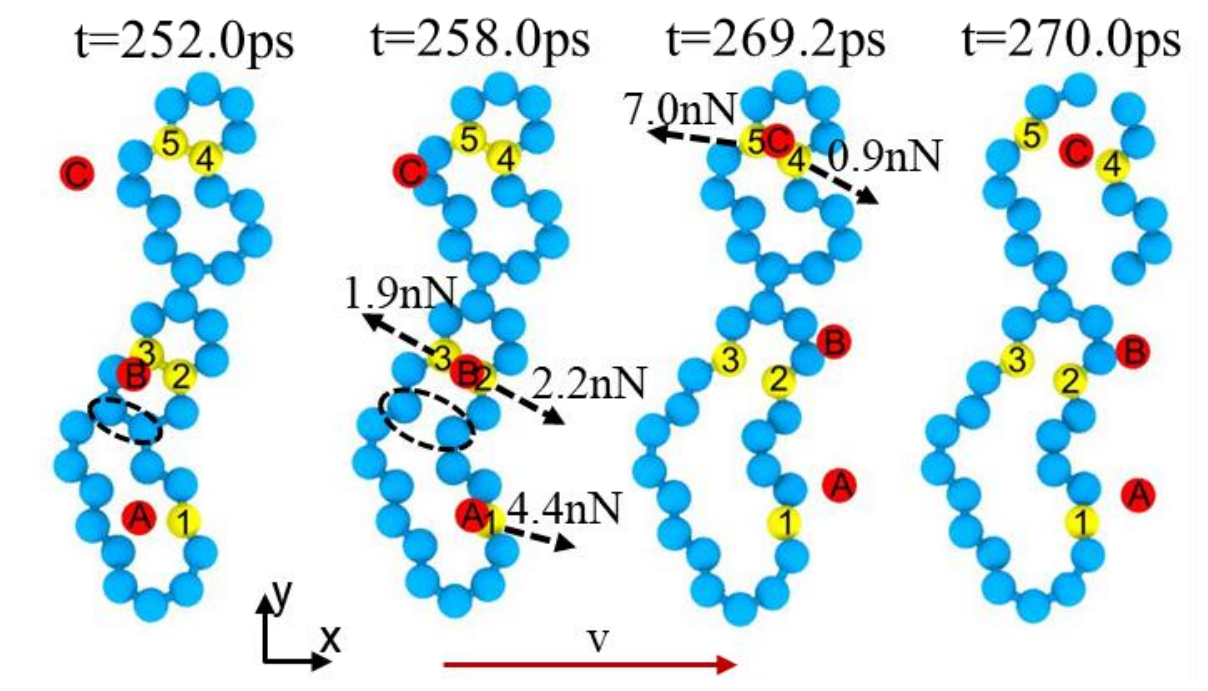

Fig.6. Snapshots of specific tip atoms (red) and carbon atoms (blue and yellow) of graphene with ZZ-132 GB during tip shearing at the load of $370 \mathrm{nN}$. Yellow color, black dotted circles and dashed arrows help track the bond-breaking. The force exerted from the specific tip atoms on yellow atoms are given.

3.3 Mitigating the weakening effects of the low-angle GBs

From the above discussions, wear resistance of the graphene layers with large-angle GBs is almost comparable to that of the pristine graphene, whereas the low-angle GBs, however, significantly deteriorate the performance of the graphene coatings. Therefore, minimizing the weakening effects of the low-angle GBs is important in order to improve graphene coating quality for potential applications. One way is to increase its interfacial adhesion to the substrate. In the above simulations, the Lennard-Jones parameters give the work of adhesion of 0.094 
$\mathrm{J} / \mathrm{m}^{2}$, which is close to $0.096 \mathrm{~J} / \mathrm{m}^{2}$ between pristine graphene and $\mathrm{SiO}_{2}$ in the experiments[47]. Here, we supplemented three additional cases with different work of adhesion, $0.039 \mathrm{~J} / \mathrm{m}^{2}, 0.23$ $\mathrm{J} / \mathrm{m}^{2}$ and $0.75 \mathrm{~J} / \mathrm{m}^{2}$ for low-angle GBs of ZZ-51. It is shown in Fig.7 that the critical normal load for wear increases with the work of adhesion between the ZZ-51 graphene and substrate. Similar effects of substrate adhesion have been observed for graphene layer with AC-179 GB. As discussed in Section 3.1, wear resistance of the GB is related to the number of the long bonds at the contact region. We counted the number of the long bonds during the scratching for different adhesion energies at the same normal load. It is demonstrated in Fig. 8a that the number of the long bonds roughly decreases with the increasing interfacial strength during the tip scratching process. This is because the higher adhesion between the ZZ-51 graphene and substrate results in the lower indenting depth of the tip as shown in Fig. $8 \mathrm{~b}$, and therefore the larger deformation of the graphene layer. The average depth of the tip for the work of adhesion of $0.75 \mathrm{~J} / \mathrm{m}^{2}$ at $300 \mathrm{nN}$ is $0.16 \mathrm{~nm}$ lower than that of $0.039 \mathrm{~J} / \mathrm{m}^{2}$ before scratching as shown in Fig.8b. Besides, the number of the long bonds increases rapidly for different adhesion energies after the tip reaches to the GB as shown in Fig. 8a, indicating that the GB is still the weak point of the graphene regardless of the interfacial strength. Another possible reason can be deduced from Fig.7, which indicates that the increase of the work of adhesion could lower the average heights of the GB buckling considerably. The higher GB buckling could be more harmful to the wear resistance of graphene. Similar findings have also been reported previously that graphene wear starts from wrinkles, as wrinkles lead to the increase of the friction forces due 
to the puckering effect [11]. Many methods have been proposed to increase the graphenesubstrate strength, such as plasma treatment [53] and deposition of graphene on mica [4].

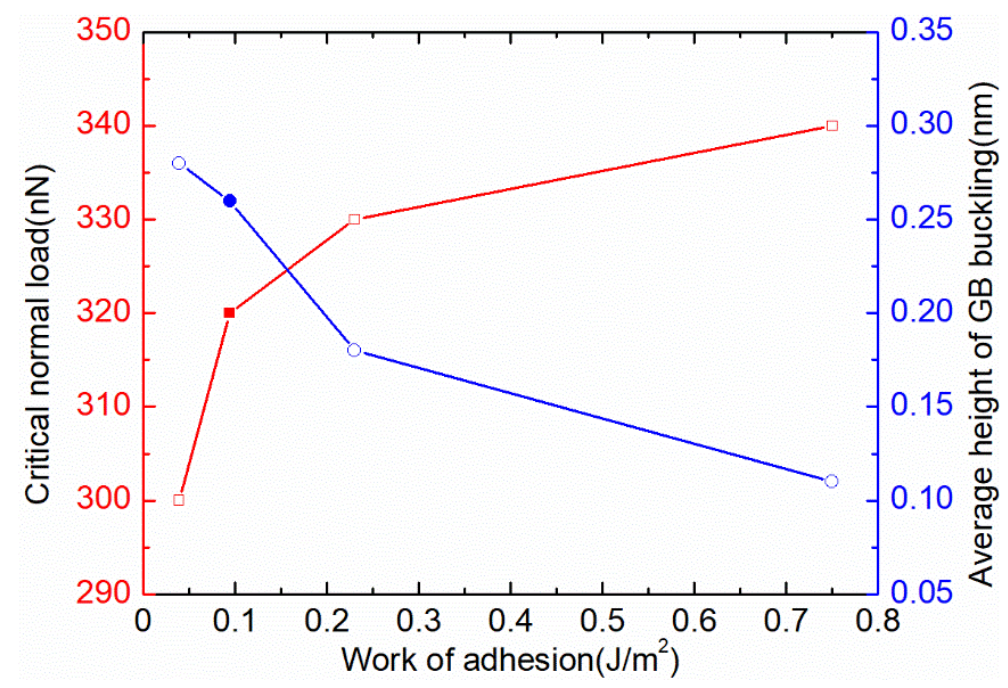

Fig.7. Critical normal load and average height of GB buckling with respect to work of adhesion between graphene with ZZ-51 and substrate.
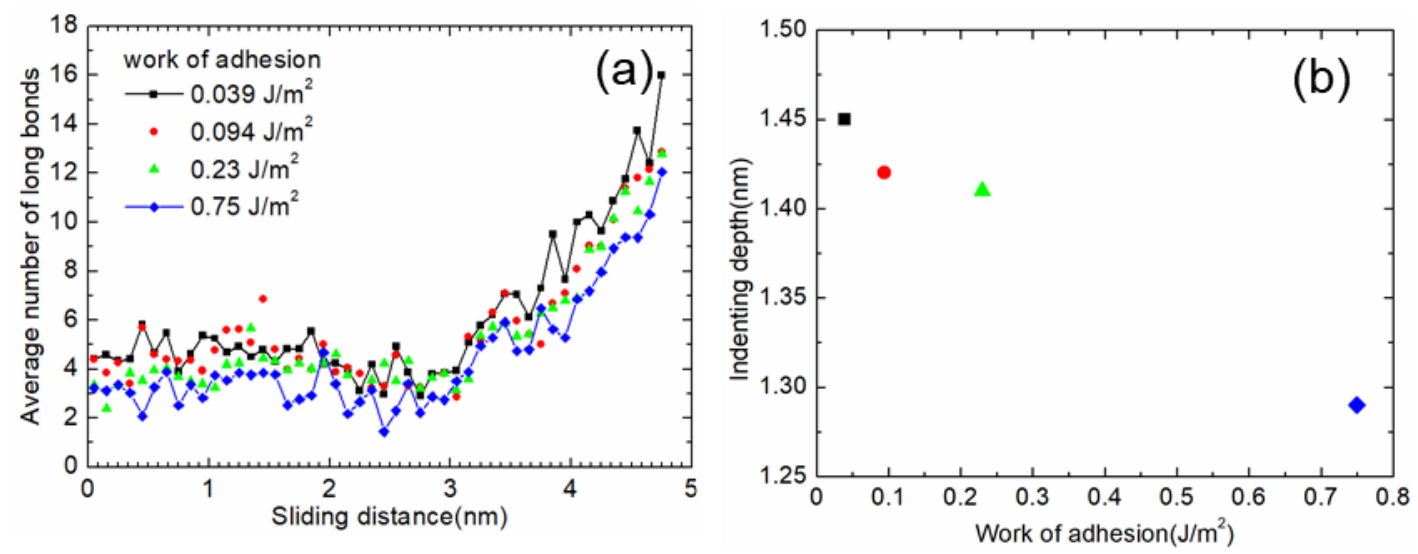

Fig. 8. (a)Variations of the long bonds in the contact region for ZZ-51 with four different adhesion $\left(0.039 \mathrm{~J} / \mathrm{m}^{2}, 0.094 \mathrm{~J} / \mathrm{m}^{2}, 0.23 \mathrm{~J} / \mathrm{m}^{2}\right.$ and $0.75 \mathrm{~J} / \mathrm{m}^{2}$.). (b) Indenting depth of the tip with respect to work of adhesion between graphene with ZZ-51 and substrate. The normal load is $300 \mathrm{nN}$. 


\section{Conclusion}

In conclusion, the wear mechanism of graphene with GBs was investigated by using largescale atomistic simulations. We manifested the synergetic actions of interlocking and pushing between the tip and graphene atoms during the scratching across the GBs, which accompany the large instantaneous force on the graphene atoms and the movement of the carbon chain both contributing to the $\mathrm{C}-\mathrm{C}$ bond breaking. Due to the lack of interfacial sliding process for pure indentation upon the GBs, the graphene with the GBs survives at much larger loads. Furthermore, wear resistance is closely related to the misorientation angle of GBs. The graphene with the low-angle GBs could lead to a significant reduction in critical normal load for wear failure as compared with pristine graphene, while wear resistance of graphene with the large-angle GBs is comparable to but slightly lower than that of pristine graphene. In contrast to the large-angle GBs, the low-angle GBs weaken wear resistance of graphene more severely owing to the presence of more long bonds in the vicinity of the GBs during the scratching process. It should be mentioned that besides the misorientation angle between the grains, the orientation of bicrystal graphene itself (e.g. armchair- or zigzag-oriented GB) also affects the wear resistance. In addition, it has been demonstrated that strengthening the interfacial interaction between the graphene and underlying substrate is a feasible method to mitigate the weakening effects on wear resistance as both buckling of the low-angle GBs and the number of the long bonds reduce. Our findings highlight the importance of controlling the microstructure, e.g. the low-angle GBs, to improve wear resistance of graphene coating. 


\section{Acknowledgement}

The authors would like to acknowledge the support of the National Natural Science Foundation of China (Grant Nos. 51527901, 51605247). Computations were carried out on the "Explorer 100" cluster system of Tsinghua National Laboratory for Information Science and Technology.

\section{References}

[1] Lee C, Wei X, Kysar JW, Hone J. Measurement of the Elastic Properties and Intrinsic Strength of Monolayer Graphene. Science. 2008;321(5887):385-8.

[2] Klemenz A, Pastewka L, Balakrishna SG, Caron A, Bennewitz R, Moseler M. Atomic Scale Mechanisms of Friction Reduction and Wear Protection by Graphene. Nano Lett. 2014;14(12):7145-52.

[3] Chen XD, Chen ZL, Sun JY, Zhang YF, Liu ZF. Graphene Glass: Direct Growth of Graphene on Traditional Glasses. Acta Phys-Chim Sin. 2011;32(1):14-27.

[4] Lee C, Li Q, Kalb W, Liu XZ, Berger H, Carpick RW, et al. Frictional characteristics of atomically thin sheets. Science. 2010;328(5974):76-80.

[5] Berman D, Erdemir A, Sumant AV. Graphene: a new emerging lubricant. Mater. Today. 2014;17(1):31 42.

[6] Gao X, Chen L, Ji L, Liu X, Li H, Zhou H, et al. Humidity-sensitive macroscopic lubrication behavior of an as-sprayed graphene oxide coating. Carbon. 2018;140:124-30.

[7] Kawai S, Benassi A, Gnecco E, Söde H, Pawlak R, Feng X, et al. Superlubricity of graphene nanoribbons on gold surfaces. Science. 2016;351(6276):957.

[8] Zheng Q, Liu Z. Experimental advances in superlubricity. Friction. 2014;2(2):182-92.

[9] Liu Y, Song A, Xu Z, Zong R, Zhang J, Yang W, et al. Interlayer Friction and Superlubricity in Single Crystalline Contact Enabled by Two-Dimensional Flake-Wrapped Atomic Force Microscope Tips. ACS Nano. 2018;12(8):7638-46.

[10] Vasić B, Matković A, Gajić R, Stanković I. Wear properties of graphene edges probed by atomic force microscopy based lateral manipulation. Carbon. 2016;107:723-32.

[11] Vasić B, Zurutuza A, Gajić R. Spatial variation of wear and electrical properties across wrinkles in chemical vapour deposition graphene. Carbon. 2016;102:304-10.

[12] Qi Y, Liu J, Zhang J, Dong Y, Li Q. Wear Resistance Limited by Step Edge Failure: The Rise and Fall of Graphene as an Atomically Thin Lubricating Material. Acs Appl. Mater. Interfaces. 2017;9(1):1099-106.

[13] Qi Y, Liu J, Dong Y, Feng X-Q, Li Q. Impacts of environments on nanoscale wear behavior of graphene: Edge passivation vs. substrate pinning. Carbon. 2018;139:59-66.

[14] Xu Q, Li X, Zhang J, Hu Y, Wang H, Ma T. Suppressing Nanoscale Wear by Graphene/Graphene Interfacial Contact Architecture: A Molecular Dynamics Study. Acs Appl. Mater. Interfaces. 
2017;9(46):40959-68.

[15] Hu X, Martini A. Atomistic simulation of the effect of roughness on nanoscale wear. Comp. Mater. Sci. 2015;102:208-12.

[16] Klemenz A, Gola A, Moseler M, Pastewka L. Contact mechanics of graphene-covered metal surfaces. Appl. Phys. Lett. 2018;112(6).

[17] Liu SW, Wang HP, Xu Q, Ma TB, Yu G, Zhang C, et al. Robust microscale superlubricity under high contact pressure enabled by graphene-coated microsphere. Nat. Commun. 2017;8:14029.

[18] Liu Y, Song A, Xu Z, Zong R, Zhang J, Yang W, et al. Interlayer Friction and Superlubricity in SingleCrystalline Contact Enabled by Two-Dimensional Flake-Wrapped Atomic Force Microscope Tips. ACS Nano. 2018.

[19] Vasić B, Matković A, Ralević U, Belić M, Gajić R. Nanoscale wear of graphene and wear protection by graphene. Carbon. 2017;120:137-44

[20] Sun X-Y, Wu R, Xia R, Chu X-H, Xu Y-J. Effects of Stone-Wales and vacancy defects in atomic-scale friction on defective graphite. Appl. Phys. Lett. 2014;104(18).

[21] Li X, Cai W, An J, Kim S, Nah J, Yang D, et al. Large-Area Synthesis of High-Quality and Uniform Graphene Films on Copper Foils. Science. 2009;324(5932):1312-4.

[22] Lee J-H, Lee EK, Joo W-J, Jang Y, Kim B-S, Lim JY, et al. Wafer-Scale Growth of Single-Crystal Monolayer Graphene on Reusable Hydrogen-Terminated Germanium. Science. 2014;344(6181):286-9.

[23] Berger C, Song Z, Li X, Wu X, Brown N, Naud C, et al. Electronic Confinement and Coherence in Patterned Epitaxial Graphene. Science. 2006;312(5777):1191-6.

[24] Lee S-M, Kim J-H, Ahn J-H. Graphene as a flexible electronic material: mechanical limitations by defect formation and efforts to overcome. Mater. Today. 2015;18(6):336-44.

[25] Kotakoski J, Meyer JC. Mechanical properties of polycrystalline graphene based on a realistic atomistic model. Phys. Rev. B. 2012;85(19):195447.

[26] Kavalur A, Kim WK. Molecular dynamics study on friction of polycrystalline graphene. Comp. Mater. Sci. 2017;137:346-61.

[27] Huang PY, Ruiz-Vargas CS, van der Zande AM, Whitney WS, Levendorf MP, Kevek JW, et al. Grains and grain boundaries in single-layer graphene atomic patchwork quilts. Nature. 2011;469(7330):389-92.

[28] Kim K, Artyukhov VI, Regan W, Liu Y, Crommie MF, Yakobson Bl, et al. Ripping Graphene: Preferred Directions. Nano Lett. 2012;12(1):293-7.

[29] Rasool HI, Ophus C, Klug WS, Zettl A, Gimzewski JK. Measurement of the intrinsic strength of crystalline and polycrystalline graphene. Nat. Commun. 2013;4(7):2811.

[30] Sha ZD, Wan Q, Pei QX, Quek SS, Liu ZS, Zhang YW, et al. On the failure load and mechanism of polycrystalline graphene by nanoindentation. Sci. Rep. 2014;4:7437.

[31] Grantab R, Shenoy VB, Ruoff RS. Anomalous Strength Characteristics of Tilt Grain Boundaries in Graphene. Science. 2010;330(6006):946.

[32] Wei Y, Wu J, Yin H, Shi X, Yang R, Dresselhaus M. The nature of strength enhancement and weakening by pentagon-heptagon defects in graphene. Nat. Mater. 2012;11(9):759-63.

[33] Yi L, Yin Z, Zhang Y, Chang T. A theoretical evaluation of the temperature and strain-rate dependent fracture strength of tilt grain boundaries in graphene. Carbon. 2013;51:373-80.

[34] Simonis P, Goffaux C, Thiry PA, Biro LP, Lambin P, Meunier V. STM study of a grain boundary in 
graphite. Surf. Sci. 2002;511(1):319-22.

[35] Ma C, Sun H, Zhao Y, Li B, Li Q, Zhao A, et al. Evidence of van Hove singularities in ordered grain boundaries of graphene. Phys. Rev. Lett. 2014;112(22):226802.

[36] Lee J-Y, Lee J-H, Kim MJ, Dash JK, Lee C-H, Joshi R, et al. Direct observation of grain boundaries in chemical vapor deposited graphene. Carbon. 2017;115:147-53.

[37] Kim K, Lee Z, Regan W, Kisielowski C, Crommie MF, Zettl A. Grain Boundary Mapping in Polycrystalline Graphene. ACS Nano. 2011;5(3):2142-6.

[38] Tison Y, Lagoute J, Repain V, Chacon C, Girard Y, Joucken F, et al. Grain boundaries in graphene on $\mathrm{SiC}(0001)$ substrate. Nano Lett. 2014;14(11):6382-6.

[39] Varchon F, Mallet P, Magaud L, Veuillen J-Y. Rotational disorder in few-layer graphene films on 6HSiC: A scanning tunneling microscopy study. Phys. Rev. B. 2008;77(16):165415.

[40] Liu T-H, Gajewski G, Pao C-W, Chang C-C. Structure, energy, and structural transformations of graphene grain boundaries from atomistic simulations. Carbon. 2011;49(7):2306 -17.

[41] Carlsson JM, Ghiringhelli LM, Fasolino A. Theory and hierarchical calculations of the structure and energetics of [0001] tilt grain boundaries in graphene. Phys. Rev. B. 2011;84(16).

[42] Pastewka L, Pou P, Pérez R, Gumbsch P, Moseler M. Describing bond-breaking processes by reactive potentials: Importance of an environment-dependent interaction range. Phys. Rev. B. 2008;78(16).

[43] Dong Y, Li Q, Martini A. Molecular dynamics simulation of atomic friction: A review and guide. J. Vac. Sci. Technol., A. 2013;31(3).

[44] Pastewka L, Klemenz A, Gumbsch P, Moseler M. Screened empirical bond-order potentials for Si-C. Phys. Rev. B. 2013;87(20).

[45] Munetoh S, Motooka T, Moriguchi K, Shintani A. Interatomic potential for Si-O systems using Tersoff parameterization. Comp. Mater. Sci. 2007;39(2):334-9.

[46] Dong $\mathrm{Y}, \mathrm{Wu} \mathrm{X}$, Martini A. Atomic roughness enhanced friction on hydrogenated graphene. Nanotechnology. 2013;24(37):375701.

[47] Ishigami M, Chen JH, Cullen WG, Fuhrer MS, Williams ED. Atomic Structure of Graphene on SiO2. Nano Lett. 2007;7(6):1643-8.

[48] Plimpton S. Fast Parallel Algorithms for Short-Range Molecular Dynamics. J. Comput. Phys. 1995;117(1):1-19.

[49] Sandoz-Rosado EJ, Tertuliano OA, Terrell EJ. An atomistic study of the abrasive wear and failure of graphene sheets when used as a solid lubricant and a comparison to diamond-like-carbon coatings. Carbon. 2012;50(11):4078-84.

[50] Egberts P, Han GH, Liu XZ, Johnson ATC, Carpick RW. Frictional Behavior of Atomically Thin Sheets: Hexagonal-Shaped Graphene Islands Grown on Copper by Chemical Vapor Deposition. ACS Nano. 2014;8(5):5010-21.

[51] Li S, Li Q, Carpick RW, Gumbsch P, Liu XZ, Ding X, et al. The evolving quality of frictional contact with graphene. Nature. 2016;539(7630):541-5.

[52] Ye Z, Balkanci A, Martini A, Baykara MZ. Effect of roughness on the layer-dependent friction of fewlayer graphene. Phys. Rev. B. 2017;96(11).

[53] Zeng X, Peng Y, Lang H. A novel approach to decrease friction of graphene. Carbon. 2017;118:23340. 
